\title{
Tfcp2l1 drives Notch signalling and epithelial diversity in the collecting duct
}

Unlike other renal epithelia, the collecting duct epithelium is formed by two different cell types: intercalated cells (IC), which regulate acid-base homeostasis, and principal cells (PC), which control salt-water equilibrium. Those two cell types function in a coordinated fashion but whether and how their development is also synchronous is unclear. Now, Jonathan Barasch and coworkers show that $T f c p 2 l 1$ coordinates IC and $\mathrm{PC}$ specification through modulation of the Jag1-Notch pathway.

To investigate how $T f c p 2 l 1$ modulates IC and PC fate, the researchers specifically ablated this transcription factor in a cell-type and stage-specific manner in the collecting duct. They showed that $T f c p 2 l 1$ is required for the formation of intermediary progenitors that express both IC and PC markers and for the maintenance of IC identity.

Tfcp2II coordinates IC and PC specification through modulation of the Jag 1-Notch pathway
By analysing RNA-Seq and ChIP-Seq data the researchers found that $T f c p 2 l 1$ positively regulates the expression of many IC-specific genes, but not PC-specific genes, including components of the Notch pathway. The ablation of $T f c p 2 l 1$ led to a complete absence of Jag1, a Notch ligand, in ICs and abolished Notch signalling in PCs. Deletion of Jag1 increased the proportion of intermediate progenitors and prevented the separation of IC and PC identities. "We showed that a single transcription factor can induce epithelia by cell-autonomous directed gene expression and, to our surprise, by non-cell autonomous gene expression via its regulation of Jag1- Notch signalling”, explains Barasch.

"Our data adds to a growing body of research highlighting the importance of grainyhead/CP2 type transcription factors in renal biology," adds Barasch. "These data should be followed by an analysis of the role of $T f c p 2 l 1$ and the Jag1-Notch pathways in innate immune functions of the collecting duct."

Andrea Aguilar

ORIGINAL ARTICLE Werth, M. et al. Transcription factor TFCP2L1 patterns cells in the mouse kidney collecting ducts. eLife 6, e24265 (2017) 